\title{
Effect of glycerol on sustained insulin release from PVA hydrogels and its application in diabetes therapy
}

\author{
YUNPENG CAI ${ }^{1,2}$, JUNYI CHE ${ }^{2}$, MINGLU YUAN ${ }^{2}$, XIAOHONG SHI $^{3 *}$, WEI CHEN $^{1^{*}}$ and WEI-EN YUAN ${ }^{2 *}$ \\ ${ }^{1}$ Department of Neurology, Xinhua Hospital Affiliated to Shanghai Jiaotong University Medical School, Shanghai 200092; \\ ${ }^{2}$ Department of Pharmaceutical Science, School of Pharmacy, Shanghai Jiaotong University, Shanghai 200240; \\ ${ }^{3}$ Department of Endocrinology, Jinshan Hospital, Fudan University, Shanghai 201508, P.R. China
}

Received June 9, 2015; Accepted June 20, 2016

DOI: $10.3892 / \mathrm{etm} .2016 .3593$

\begin{abstract}
The present study aimed to investigate the effects of glycerol on the physical properties and release of an insulin-loaded polyvinyl alcohol (PVA) hydrogel film. The insulin-loaded hydrogel composite film was produced using the freeze-thawing method, after which the in vitro swelling ratio, transmittance and insulin release, and the in vivo pharmacodynamics, of hydrogels containing various volumes of glycerol were investigated. The results demonstrated that the addition of glycerol reduced the swelling ratio and increased the softness of the PVA hydrogel film. An analysis of insulin release in vitro and of the hypoglycemic effects in rats demonstrated that the PVA hydrogel film had a sustained release of insulin and long-acting effect over 10 days. The results of the present study suggested that, as a hydrophilic plasticizer, glycerol was able to enhance the release of insulin in the early stage of release profile by enhancing the formation of water channels, although the total swelling ratio was decreased. Therefore, the insulin-loaded glycerol/PVA hydrogel film may be a promising sustained-release preparation for the treatment of diabetes.
\end{abstract}

\section{Introduction}

Hydrogels have a three-dimensional network that consists of hydrophilic polymers that are able to absorb a large volume of water and swell, instead of undergoing dissolution (1). Hydrogels are soft and able to maintain a certain shape. All water-soluble

Correspondence to: Dr Xiaohong Shi, Department of Endocrinology, Jinshan Hospital, Fudan University, 1508 Longhang Road, Shanghai 201508, P.R. China

E-mail: shixh80301@163.com

Dr Wei Chen, Department of Neurology, Xinhua Hospital Affiliated to Shanghai Jiaotong University Medical School, 1665 Kongjiang Road, Shanghai 200092, P.R. China

E-mail: chen_wei3000@126.com

${ }^{*}$ Contributed equally

Key words: hydrogel, polyvinyl alcohol, insulin, sustained release or hydrophilic polymers are able to form a hydrogel via chemical or physical cross-linking (2). Due to their unique physicochemical properties and biocompatibility, hydrogels are widely used in multiple fields, including cellular immobilization, tissue engineering and regenerative medicine, diagnostics, separation of biomolecules or cells and wound dressing (3-7). It has been reported that insulin is able to accelerate wound healing by reducing inflammation and increasing collagen deposition $(8,9)$. Furthermore, hydrogels have been extensively investigated as drug delivery systems for sustained release (10).

Wichterle and Lim (11) first reported the potential for using hydrogels in medical research in 1960. Since then, research into the design and preparation of polymer hydrogels has increased (11). Polyvinyl alcohol (PVA) is frequently employed due to its biocompatibility and biodegradability (12-14). In addition, PVA hydrogels have a high water content and high strength, and are easily produced $(15,16)$. In order to prepare hydrogels, various cross-linking strategies are used, including chemical and physical cross-linking. However, chemical cross-linking requires initiators, such as glutaraldehyde, diisocyanates or acyl azide, whose inherent cytotoxicity may reduce the biocompatibility of the hydrogel (16). Furthermore, cross-linking agents may lead to further challenges in the purification of the final product (17). In the present study, the simple and physical freeze-thawing method was used to produce PVA hydrogels, in order to avoid detrimental factors that have been associated with chemical cross-linking.

Glycerol, which is generated by the hydrolysis of triglycerides, is predominantly produced in the process of saponification or biodiesel production (18). It occurs as a clear, colorless, odorless, viscous and hygroscopic liquid with a sweet taste (19). Glycerol has become increasingly attractive due to its wide range of applications in the food industry (plasticizer, stabilizer and emulsifier) and as a humectant in cosmetic formulations (20-23). In addition, it has been shown to have an important role as a plasticizer for the generation of hyper-branched structures (24-26). However, few previous studies have investigated the use of glycerol as a cross-linking agent to adjust the density of a polymeric network in order to achieve sustained release behavior.

The present study aimed to investigate the effects of glycerol on the in vitro and in vivo properties of a PVA hydrogel film, in particular on its in vitro mechanical, swelling, transmittance and release properties, and in vivo hypoglycemic activity. 


\section{Materials and methods}

Animals and reagents. Insulin (28 IU/mg) was purchased from Gan \& Lee Pharmaceutical (Beijing, China). Analytically pure PVA (110 kDa, 98\% hydrolyzed), ethanolamine, glycerol, phosphoric acid, urethane and disodium hydrogen phosphate were purchased from Sinopharm Chemical Reagent Co., Ltd. (Shanghai, China). A total of 25 male Wistar diabetic rats weighing $200 \pm 20 \mathrm{~g}$ and aged 4 weeks were purchased from Xinhua Hospital Affiliated to Shanghai Jiao Tong University School of Medicine (Shanghai, China). Protocols involving the rats were approved by the Institutional Review Board of Xinhua Hospital and were performed according to the guidelines outlined by the National Institutes of Health for the Care and Use of Laboratory Animals.

Preparation of the PVA hydrogel film. PVA $(18 \% \mathrm{w} / \mathrm{w})$ was weighed and dissolved in boiling water with constant stirring to form a solution. The solution was cooled to room temperature, after which a certain amount of insulin (14.2 mg, 1\% of PVA weight) was added under vortex. Various amounts of glycerol $(0,50,100,150$ and $200 \mu \mathrm{l})$ were subsequently added and mixed, followed by loading of the solution onto a glass plate and pressing with another glass plate. Plates were frozen at $-20^{\circ} \mathrm{C}$ for $2 \mathrm{~h}$, and subsequently defrosted at $4^{\circ} \mathrm{C}$ for $1 \mathrm{~h}$. The freeze-thaw cycle was repeated various times to obtain hydrogels with different mechanical properties, as described previously (27). Hydrogel films were dried in a vacuum dryer at room temperature for $24 \mathrm{~h}$. Fig. 1 shows the respective chemical structures of PVA and glycerol.

Swelling ratio. PVA hydrogel films of 1-mm thickness were cut into discs with a diameter of $12 \mathrm{~mm}$ and subsequently weighed. Samples were immersed in distilled water at room temperature, prior to drying and weighing every 30 min until a constant weight was achieved. The swelling ratio was defined using the following equation:

$$
\text { Swelling ratio }(\%)=\frac{m_{1}-m_{0}}{m_{0}} \times 100 \%
$$

Where $\mathrm{m}_{0}$ is the original weight $(\mathrm{g})$ and $\mathrm{m}_{1}$ is the weight $(\mathrm{g})$ following immersion.

Transmittance. PVA hydrogel films of 1-mm thickness were cut into rectangular shapes $(5 \times 20 \mathrm{~mm})$ and attached to the surface of cuvettes. Absorbance was read at $500 \mathrm{~nm}$ using a spectrophotometer and the transmittance was calculated by comparing with the cuvette-only group, as described previously (28).

Insulin release in vitro. Dry films were cut into discs with a diameter of $12 \mathrm{~mm}$. Phosphate-buffered saline (PBS) solution containing $8.5 \mathrm{~g}$ sodium chloride, $5.8 \mathrm{~g}$ disodium hydrogen phosphate dodecahydrate, $0.6 \mathrm{~g}$ sodium dihydrogen phosphate and $11 \mathrm{H}_{2} \mathrm{O}(\mathrm{pH}$ 7.4) was prepared for use as the release medium buffer. A 6-well plate was used as the container, and hydrogel films released insulin into a water bath at $37^{\circ} \mathrm{C}$. To ensure only one side of the film was in contact with the PBS, wire meshes were used to separate the hydrogel film from the PBS. For each
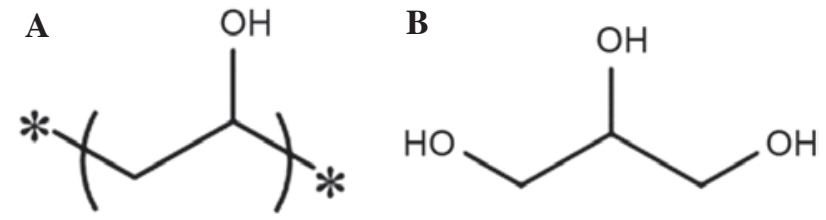

Figure 1. Chemical structures of (A) polyvinyl alcohol and (B) glycerol.

well, $5 \mathrm{ml}$ PBS was added and covered with a wire mesh, after which PVA hydrogel films were placed onto the wire mesh. At certain time points $(0.5,1,2,4,6,8,10,24,36,48$ and $60 \mathrm{~h})$, the PBS solutions were replaced with fresh PBS solution of the same volume. Following $60 \mathrm{~h}, 5 \mathrm{ml}$ phosphoric acid ( $5 \mathrm{~mol} / \mathrm{l})$ was added and the plates were incubated at $37^{\circ} \mathrm{C}$ overnight to dissolve all the remaining insulin.

The amount of insulin in the respective solutions was measured by high performance liquid chromatography (Agilent Technologies, Inc., Santa Clara, CA, USA). For preparation of the mobile phase, $21.06 \mathrm{~g}$ sodium sulfate, $2 \mathrm{ml}$ phosphoric acid and $222 \mu \mathrm{l}$ ethanolamine were dissolved in $740 \mathrm{ml}$ distilled water. The stationary phase consisted of a carbon-18 250-mm column. A flow rate of $1 \mathrm{ml} / \mathrm{min}$, an injection volume of $80 \mu \mathrm{l}$ and a detection wavelength of $214 \mathrm{~nm}$ were used, as described previously (29).

Hypoglycemic effect in rats. Hydrogel films containing 12 IU insulin were prepared by six freeze-thaw cycles and cut into discs with a diameter of $12 \mathrm{~mm}$. A total of 25 male Wistar rats (5 weeks old) were used throughout the study. The animals were raised in rooms controlled at $23 \pm 1^{\circ} \mathrm{C}$ and $55 \pm 5 \%$ relative humidity with a 12 -h light/dark cycle. They received standard laboratory chow diet and tap water during acclimatization. Rats were anaesthetized by injection with $5 \mathrm{ml}(20 \% \mathrm{w} / \mathrm{v})$ urethane aqueous solution, after which insulin-loaded or blank PVA hydrogel films, or insulin-loaded PVA hydrogel films containing $50,100,150$ or $200 \mu \mathrm{l}$ glycerol, were implanted under the dorsal skin of the rats. Blood samples $(200 \mu \mathrm{l})$ were collected from the rat tail veins at various time intervals $(4,10,20,30,40,50$, $60,70,120,150,200$ and $240 \mathrm{~h}$ ) for blood glucose assays. The concentration of blood glucose was determined using a glucometer (Roche Diagnostics, GmbH, Mannheim, Germany). Rats were sacrificed by cervical dislocation under mild anesthesia using pentobarbital $(50 \mathrm{mg} / \mathrm{kg})$ after this experiment.

Statistical analysis. The data from different groups were presented as the mean \pm standard deviation. Differences between groups were analyzed using Student's test by Excel 2010 and statistical significance was set at $\mathrm{P}<0.05$.

\section{Results and Discussion}

Transmittance. Table I shows the transmittance of the PVA hydrogels containing various quantities of glycerol. The crystallinity of PVA is an important physical property for measuring the degree of cross-linking (30). PVA crystalline regions are able to scatter and reflect light, thereby lowering the opacity of the hydrogel $(31,32)$. The present study indirectly measured the degree of crystallinity of the hydrogels by investigating the transmittance. Transmittance of the PVA hydrogel decreased 
Table I. Transmittance of PVA hydrogel containing various amounts of glycerol.

\begin{tabular}{lc} 
Glycerol $(\mu 1)$ & Transmittance $(\%)$ \\
\hline 0 & 77.1 \\
50 & 75.9 \\
100 & 74.2 \\
150 & 73.6 \\
200 & 69.2 \\
\hline
\end{tabular}

The hydrogel formulation consisted of $1.426 \mathrm{~g}$ PVA dissolved in $6.56 \mathrm{ml}$ water and various volumes of glycerol. PVA, polyvinyl alcohol.

as the concentration of glycerol increased. A transmittance of $69.2 \%$ was attained with $3 \%(\mathrm{v} / \mathrm{v} ; 200 \mu \mathrm{l})$ glycerol.

Hydrogels prepared by irradiation have a higher transparency, as compared with those prepared by freeze-thawing; however, irradiation-prepared hydrogels typically have a poor mechanical strength due to the lack of physical cross-linking in crystallites that are introduced by freeze-thawing (33). However, the addition of glycerol should decrease the crystallinity of PVA molecules, since it is able to insert into the polymeric chains and disturb the order of chains, resulting in a higher transmittance (32). Therefore, the relatively high transmittance observed in the present study may be attributed to the formation of new crosslinks between the PVA and glycerol molecules. The formulation containing $200 \mu l$ glycerol was considered to be the optimum, since it showed higher crosslinking degree indicated by the transmittance and improved softness.

Swelling ratio. Swelling behavior is an important parameter that is indicative of the water absorbing capacity of a hydrogel (34). The films began to swell immediately upon immersion, and the swelling speed increased markedly from 10-60 min. After $60 \mathrm{~min}$, the swelling rate decreased and swelling finally terminated after $180 \mathrm{~min}$. Microscopic observations demonstrated the appearance of pores on the film following immersion, which served as the channels for water and drug release (Fig. 2). Fig. 3 shows the swelling ratio of hydrofilms containing various glycerol amounts. The swelling ratio was shown to decrease as the glycerol content increased, although the difference between the various glycerol contents was not significant (Fig. 3).

Glycerol molecules are able to occupy the space surrounding the hydroxyl groups of PVA and may form crosslinks between PVA molecules; thus they may restrict the number of contacts made between PVA and water molecules. Since water acts as a channel for drug diffusion, an increased sustained-release effect may be obtained by reducing the swelling ratio $(33,35,36)$.

Insulin release in vitro. Fig. 4 shows the release curves for PVA hydrogels that underwent various freeze-thaw cycles. The freeze-thaw method is a physical method used to crosslink the polymers, and the number of cycles may have a significant effect on the release behavior of the hydrogel (37). A previous study reported that the cross-linking characteristics and drug release properties of hydrogels may be optimized by controlling the number of cycles and duration of freeze/thawing (38). As

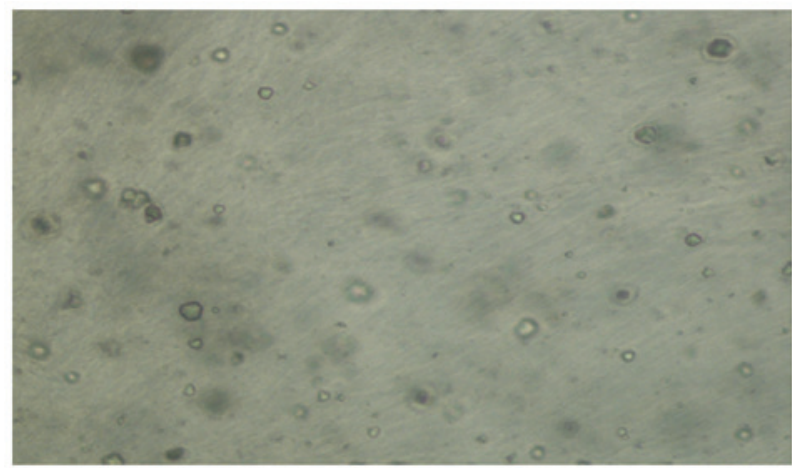

Figure 2. Microscopic observation of the holes on the surface of the polyvinyl alcohol membrane structure (magnification, $\mathrm{x} 40$ ).

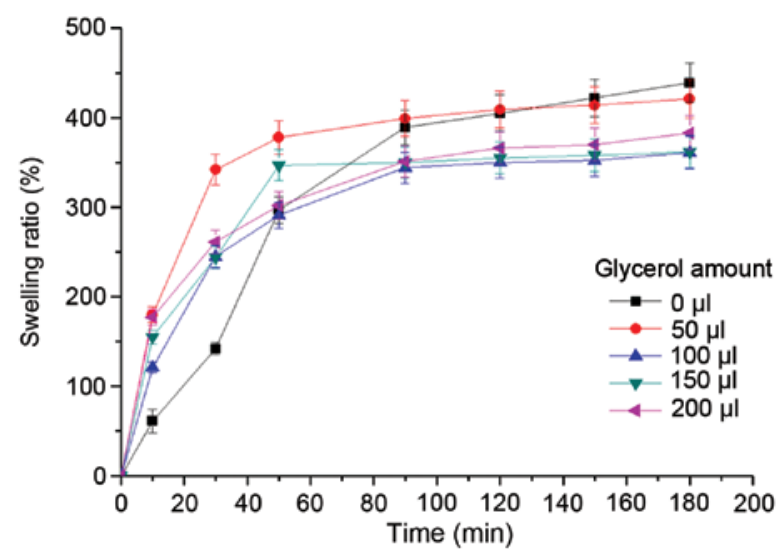

Figure 3. Swelling ratio of polyvinyl alcohol hydrogels containing various amounts of glycerol. Data are presented as the mean \pm standard deviation $(n=3)$.

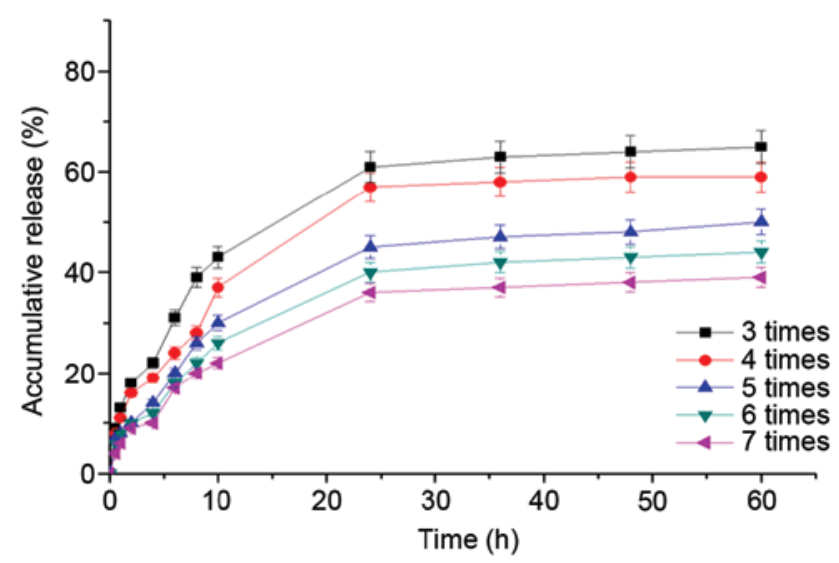

Figure 4. In vitro release curves of polyvinyl alcohol hydrogels that underwent 3-7 freeze-thaw cycles. Data are presented as the mean \pm standard deviation, $\mathrm{n}=3$.

is shown in Fig. 4, the number of freeze-thaw repetitions had a marked effect on the release rate and total release amount. The total release amount decreased from 66 to $38 \%$ as the number of freeze-thaw repetitions increased. Since freeze-thawing is a physical cross-linking method, the density of crystalline regions is increased as the number of freeze-thaw cycles is increased $(30,39)$. The release of insulin is matrix-controlled, 
therefore insulin is not released until the polymer is sufficiently degraded (40). Hence, a highly crosslinked structure is associated with a slower release, and drugs may not be released completely (40). Furthermore, our unpublished study demonstrated that the release of insulin was too fast when the hydrogel film was totally immersed in water. Considering that only one side of the hydrogel would be in contact with body fluid in vivo, a mesh was used in the present study to ensure that only one side was in contact with the release medium.

As is shown in Fig. 5, glycerol was able to enhance the release of insulin in vitro. Fig. 5 shows the in vitro release curves for PVA hydrogels containing various amounts of glycerol. Higher volumes of glycerol accelerated the release of insulin and increased the total release amount, as compared with the control group. Accumulative release was increased from 41.1 to $49.7 \%$ and the release was increased from 23.3 to $35.1 \%$ in the initial $10 \mathrm{~h}$. Although glycerol may form physical crosslinks with PVA molecules and decrease the absorption of water, we hypothesize that the hydrophilic property of glycerol may compensate for the slow release due to less water. It may be that glycerol was able to insert into the PVA chains and enlarge the channels for insulin release when combined with water. However, although higher volumes of glycerol were able to increase the total release, the mechanical properties of hydrogels with higher glycerol contents were unacceptable. Hydrogels that are too soft do not maintain their shape and the resulting leakage of glycerol and drugs poses potential risks, e.g., the unexpected drug burst release induce overdose risk.

Hypoglycemic effect. Fig. 6 shows the blood glucose concentration in diabetic rats implanted with insulin-loaded or blank PVA hydrogel films. The blood glucose concentration of the blank control group was normal and stable; it fluctuated within a narrow range of 23-28 mmol/l. The experimental group treated with $12 \mathrm{IU}$ insulin exhibited a rapid decrease in blood glucose concentration, which lasted for 10 days. Following administration, the most obvious difference was observed between days 0 and 6 , in which the mean blood glucose level reduced by $>50 \%$. After 6 days, the experimental group maintained a marginally lower blood glucose level, as compared with the control group, thus suggesting that insulin was still being released at a low rate. These results suggested that the release of insulin had changed from the swelling-controlled release in the early stage to matrix-controlled release in the later stage.

The hypoglycemic effect lasted longer, as compared with the release period in vitro. This may have been due to the PVA hydrogel not swelling as completely and as quickly in vivo, as compared with the PBS solution in vitro. The absorption of water is an important factor that regulates the early stage release, and has a marked influence on the pore-opening and channel-forming processes (41). A previous study demonstrated that the release of insulin from the hydrogel was dependent on diffusion through the water hydrating the polymer network (42). In the present study, if the film had not been implanted under the dorsal skin, but instead used to cover the wound as one of the applications of insulin (9), the release may have been even slower.

As is shown in Fig. 5, the release of insulin was also affected by the amount of glycerol, which was consistent with the in vitro data. Fig. 7 shows the blood glucose concentrations of rats

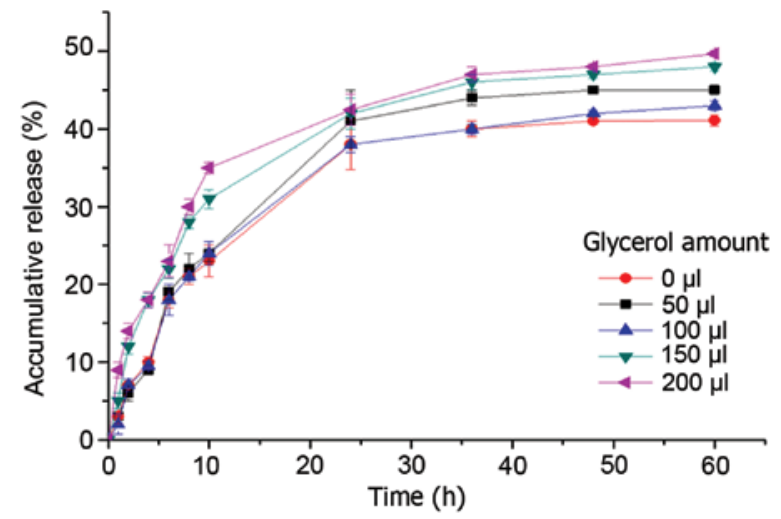

Figure 5. In vitro release curves of polyvinyl alcohol hydrogels containing various amounts of glycerol. Data are presented as the mean \pm standard deviation, $\mathrm{n}=3$.

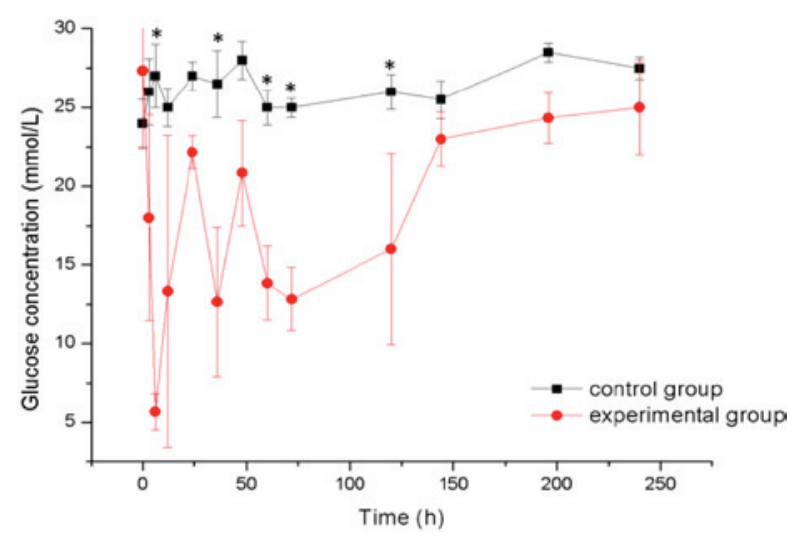

Figure 6. Blood glucose concentrations of diabetic rats implanted with insulin-loaded or blank polyvinyl alcohol (PVA) hydrogel films. The experimental group was implanted with a PVA hydrogel film that contained $12 \mathrm{IU}$ insulin and $200 \mu \mathrm{l}$ glycerol. After $6 \mathrm{~h}$, an injection of $2 \mathrm{ml}$ glucose solution (5\%) was administered to prevent death due to hypoglycemia. Data are presented as the mean \pm standard deviation, $n=5\left({ }^{*} \mathrm{P}<0.05\right)$.

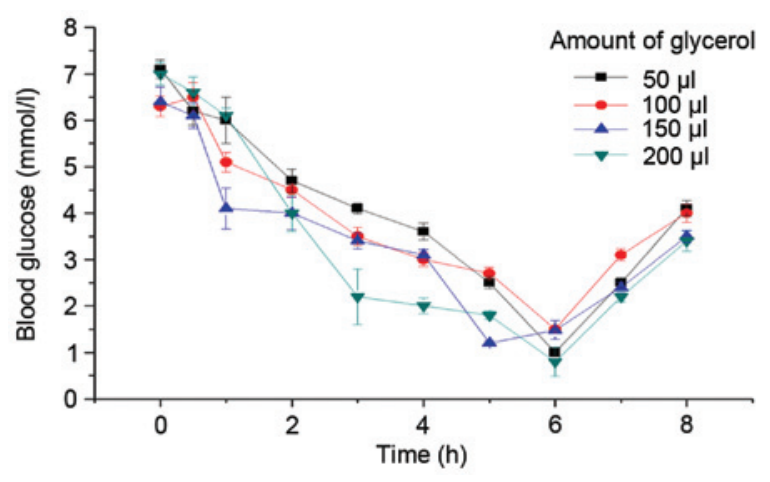

Figure 7. Blood glucose concentrations of diabetic rats implanted with polyvinyl alcohol hydrogel films containing various amounts of glycerol. Data are presented as the mean \pm standard deviation, $n=5$. In this experiment, the rats did not receive a glucose supply and succumbed to hypoglycemia after $8 \mathrm{~h}$.

implanted with PVA hydrogels containing various amounts of glycerol. Notably, the higher the glycerol content, the quicker the insulin was released and the more rapid the decrease in blood glucose concentration. In the initial $6 \mathrm{~h}$, the blood glucose concentration was rapidly decreased, after which it began to 
recover. Although the present data does not include time points after $8 \mathrm{~h}$, the correlation between the in vitro and in vivo data and influence of glycerol were clearly demonstrated. These results suggested that blood glucose concentrations were decreased to a greater extent with higher amounts of glycerol.

In the present study, glycerol was used to adjust the mechanical property and release behavior of a PVA hydrogel. In addition, the transmittance, swelling ratio, release in vitro and in vivo hypoglycemic effect of PVA hydogel films containing various amount of glycerol were investigated. The results demonstrated that the addition of glycerol reduced the swelling ratio and hardness of the hydrogel, and enhanced the release of insulin in vitro and in vivo. Although the early phase of release was shown to be swelling-controlled and glycerol was shown to lower the swelling ratio, as a hydrophilic plasticizer, glycerol was able to accelerate the release in the early stage, potentially due to its enhanced ability of forming channels for water, as compared with PVA. The insulin-loaded PVA hydrogel film exhibited a hypoglycemic effect in diabetic rats over 10 days.

In conclusion, the results of the present study suggested that glycerol was able to disrupt the crystallite structure of PVA molecules while forming crosslinked structures between them, thereby promoting insulin release. The improved physical properties and verified long-acting effect demonstrated the efficacy of using glycerol in PVA hydrogels for the management of diabetes. Further research is required in order to validate the wound healing effect in animal models and elucidate specific mechanical parameters (43). This study indicated the potential for using glycerol contained PVA hydrogel as the sustained release matrix for diabetes treatment, as it enhanced the insulin release and has a smooth release profile.

\section{Acknowledgements}

The present study was supported by the Interdisciplinary Projects of Medicine and Engineering of Shanghai Jiao Tong University (grant nos. YG2013MS52, YG2014QN06 and YG2013MS62).

\section{References}

1. Peppas NA, Bures P, Leobandung W and Ichikawa H: Hydrogels in pharmaceutical formulations. Eur J Pharm Biopharm 50: 27-46, 2000.

2. Hoffman AS. Hydrogels for biomedical applications. Adv Drug Deliver Rev 64: 18-23, 2012.

3. Jen AC, Wake MC and Mikos AG: Review: Hydrogels for cell immobilization. Biotechnol Bioeng 50: 357-364, 1996.

4. Lee KY and Mooney DJ: Hydrogels for tissue engineering. Chem Rev 101: 1869-1879, 2001.

5. van der Linden HJ, Herber S, Olthuis $W$ and Bergveld $P$ Stimulus-sensitive hydrogels and their applications in chemical (micro) analysis. Analyst 128: 325-331, 2003.

6. Wang K, Burban J and Cussler E: Hydrogels as separation agents. In: Responsive Gels: Volume Transitions II. Dusek K (ed). Springer-Verlag, Berlin, pp67-79, 1993.

7. Yoshii F, Zhanshan Y, Isobe K, Shinozaki K and Makuuchi K: Electron beam crosslinked PEO and PEO/PVA hydrogels for wound dressing. Radiat Phys Chem Oxf Engl 1993 55: 133-138, 1999.

8. Madibally SV, Solomon V, Mitchell RN, Van De Water L, Yarmush ML and Toner M: Influence of insulin therapy on burn wound healing in rats. J Surg Res 109: 92-100, 2003.

9. Pierre EJ, Barrow RE, Hawkins HK, Nguyen TT, Sakurai Y, Desai M, Wolfe RR and Herndon DN: Effects of insulin on wound healing. J Trauma 44: 342-345, 1998.
10. Qiu Y and Park K: Environment-sensitive hydrogels for drug delivery. Adv Drug Delivery Rev 53: 321-339, 2001.

11. Wichterle O and Lim D: Hydrophilic Gels for Biological Use. Nature 185: 117-118, 1960

12. Nho YC, Lim YM, Gwon HJ and Choi EK: Preparation and characterization of PVA/PVP/glycerin/antibacterial agent hydrogels using $\gamma$-irradiation followed by freeze-thawing. Korean J Chem Eng 26: 1675-1678, 2009.

13. Hirai T, Okinaka T, Hayashi S, Amemiya Y, Kobayashi K and Hirai M: PH-induced structure change of poly (vinyl alcohol) hydrogel crosslinked with poly (acrylic acid). Macromol Mater Eng 240: 213-219, 1996.

14. Noguchi T, Yamamuro T, Oka M, Kumar P, Kotoura Y, Hyon S and Ikada Y: Poly (vinyl alcohol) hydrogel as an artificial articular cartilage: Evaluation of biocompatibility. J Appl Biomater 2: 101-107, 1991.

15. Hyon SH, Cha WI and Ikada Y: Preparation of transparent poly (vinyl alcohol) hydrogel. Polym Bull 22: 119-122, 1989.

16. Cha WI, Hyon SH and Ikada Y: Transparent poly(vinyl alcohol) hydrogel with high water content and high strength. Die Makromol Chem 193: 1913-1925, 1992.

17. Roberts GA and Taylor KE: Chitosan gels, 3. The formation of gels by reaction of chitosan with glutaraldehyde. Macromol Mater Eng 190: 951-960, 1989.

18. Tan HW, Aziz ARA and Aroua MK: Glycerol production and its applications as a raw material: A review. Renew Sust Energ Rev 27: 118-127, 2013.

19. Allen Jr LV: Tiagabine Hydrochloride $1 \mathrm{mg} / \mathrm{ml}$ Oral Liquid. US Pharm 36: 72-73, 2011

20. Anker $M$ and Stading M: Aging of whey protein films and the effect on mechanical and barrier properties. J Agric Food Chem 49: 989-995, 2001.

21. Brougham M and Johnson D: Glycerol, $\alpha$-glycerophosphate and other compounds as stabilizers of alcohol dehydrogenase from yeast. Enzyme Microb Technol 3: 225-228, 1981.

22. Piao J and Adachi S: Stability of $\mathrm{O} / \mathrm{W}$ emulsions prepared using various monoacyl sugar alcohols as an emulsifier. Innov Food Sci Emerg Technol 7: 211-216, 2006.

23. Pedersen LK and Jemec GB: Plasticising effect of water and glycerin on human skin in vivo. J Dermatol Sci 19: 48-52, 1999.

24. Fang Y, Yu H, Chen L and Chen S: Facile glycerol-assisted synthesis of N-vinyl pyrrolidinone-based thermosensitive hydrogels via frontal polymerization. Chem Mater 21: 4711-4718, 2009.

25. Lavorgna M, Piscitelli F, Mangiacapra P and Buonocore GG: Study of the combined effect of both clay and glycerol plasticizer on the properties of chitosan films. Carbohydr Polym 82: 291-298, 2010.

26. Zhou H, Steinhilber D, Schlaad H, Sisson AL and Haag R: Glycerol based polyether-nanogels with tunable properties via acid-catalyzed epoxide-opening in miniemulsion. React Funct Polym 3: 356-361, 2011.

27. Willcox PJ, Howie DW Jr, Schmidt-Rohr K, Hoagland DA, Gido SP, Pudjijanto S, Kleiner LW and Venkatraman S: Microstructure of poly (vinyl alcohol) hydrogels produced by freeze/thaw cycling. J Polym Sci B Polym Phys 37: 3438-3454, 1999.

28. Bakhshandeh H, Soleimani M, Hosseini SS, Hashemi H, Shabani I, Shafiee A, Nejad AH, Erfan M, Dinarvand R and Atyabi F: Poly (epsilon-caprolactone) nanofibrous ring surrounding a polyvinyl alcohol hydrogel for the development of a biocompatible two-part artificial cornea. Inte J Nanomedicine 6: 1509-1515, 2011.

29. You Y, Zhao M, Liu G and Tang X: Physical characteristics and aerosolization performance of insulin dry powders for inhalation prepared by a spray drying method. J Pharm Pharmacol 59: 927-934, 2007.

30. Hassan CM and Peppas NA: Structure and morphology of freeze/thawed PVA hydrogels. Macromolecules 33: 2472-2479, 2000.

31. Mardare D, Tasca M, Delibas M and Rusu GI: On the structural properties and optical transmittance of $\mathrm{TiO}_{2}$ r.f. sputtered thin films. Appl Surf Sci 156: 200-206, 2000.

32. Huh KM and Bae YH: Synthesis and characterization of poly(ethylene glycol)/poly (L-lactic acid) alternating multiblock copolymers. Polymer 40: 6147-6155, 1999.

33. Yang X, Yang K, Wu S, Chen X, Yu F, Li J, Ma M and Zhu Z: Cytotoxicity and wound healing properties of PVA/ws-chitosan/glycerol hydrogels made by irradiation followed by freeze-thawing. Radiat Phys Chem Oxf Engl 1993 79: 606-611, 2010.

34. Kim CJ and Lee PI: Composite poly (vinyl alcohol) beads for controlled drug delivery. Pharm Res 9: 10-16, 1992. 
35. Qu X, Wirsen A and Albertsson AC: Novel pH-sensitive chitosan hydrogels: Swelling behavior and states of water. Polymer 41: 4589-4598, 2000.

36. Korsmeyer RW and Peppas NA: Effect of the morphology of hydrophilic polymeric matrices on the diffusion and release of water soluble drugs. J Memb Sci 90: 211-227, 1981.

37. Liu Y, Geever LM, Kennedy JE, Higginbotham CL, Cahill PA and McGuinness GB: Thermal behavior and mechanical properties of physically crosslinked PVA/Gelatin hydrogels. J Mech Behav Biomed 3: 203-209, 2010

38. Peppas NA and Mongia NK: Ultrapure poly (vinyl alcohol) hydrogels with mucoadhesive drug delivery characteristics. Euro J Pharm Biopharm 43: 51-58, 1997.

39. Gu ZQ, Xiao JM and Zhang XH: The development of artificial articular cartilage-PVA-hydrogel. Biomed Mater Eng 8: 75-81, 1998.
40. Huang $X$ and Brazel CS: On the importance and mechanisms of burst release in matrix-controlled drug delivery systems. J Control Release 73: 121-136, 2001.

41. Hawkins AM, Milbrandt TA, Puleo DA and Hilt JZ: Composite hydrogel scaffolds with controlled pore opening via biodegradable hydrogel porogen degradation. J Biomed Mater Res A 102: 400-412, 2014.

42. Burczak K, Fujisato T, Hatada M and Ikada Y: Protein permeation through poly (vinyl alcohol) hydrogel membranes. Biomaterials 15: 231-238, 1994.

43. Stammen JA, Williams S, Ku DN and Guldberg RE: Mechanical properties of a novel PVA hydrogel in shear and unconfined compression. Biomaterials 22: 799-806, 2001. 